\title{
Assessment of method agreement between two minimally invasive hemodynamic measurements in septic shock patients on high doses of vasopressor drugs. A preliminary study
}

\author{
Oana Antal $^{1,2}$, Mihai Mărginean ${ }^{1,3}$, Natalia Hagău ${ }^{1,2}$ \\ ${ }^{1}$ University of Medicine and Pharmacy "Iuliu Hațieganu" Cluj-Napoca, Romania \\ ${ }^{2}$ Emergency Clinical County Hospital Cluj-Napoca, Romania \\ ${ }^{3}$ Emergency Military Hospital “Dr. Constantin Papilian” Cluj-Napoca, Romania
}

\begin{abstract}
Background: Minimally invasive hemodynamic monitoring is still controversial among the methods used to assess the hemodynamic profile of the septic shock patient. The aim of this study was to test the level of agreement between two different devices.

Methods. We collected 385 data entries during 12-hour intervals from four critically ill patients with septic shock and high doses of vasoactive therapy using two minimally invasive methods at the same time: Vigileo $^{\mathrm{TM}}$ device which uses the pulse contour principle, and EV1000 ${ }^{\mathrm{TM}}$ monitoring platform which uses the transpulmonary thermodilution principle. The studied parameters were Stroke Volume (SV), Cardiac Output (CO) and Mean Arterial Pressure (MAP). We tested the agreement by performing the visual examination of data patterns using graphs and studying the bias, limits of agreement and creating BlandAltman plots. For assessing the systematic, proportional and random differences, we computed a PassingBablock regression with the CUSUM test for linearity.

Results. The one sample t-Test for the differences between the two methods against the null value was statistically significant for the studied parameters $(\mathrm{p}<0.0001)$. The Bland-Altman analysis found no agreement between the data obtained using the two techniques, with calculated error percent as high as $88.28 \%$ for SV, $82.02 \%$ for CO and $42.06 \%$ for MAP. The Passing-Bablock regression analysis tested positive for systematic differences, but this could not be accounted for.

Conclusion. We found no agreement between data obtained from the studied devices; therefore, these cannot be used interchangeably for critically ill septic shock patients on high doses of vasoactive substances.

Keywords: hemodynamic monitoring, pulse contour, transpulmonary thermodilution, septic shock patient

\section{Introduction}

Severe sepsis and septic shock are the main cause of mortality in critically ill patients [1]. Recognizing the early signs of sepsis and the different stages of this condition may lower the associated morbidity and mortality. Due to the heterogeneity of the population

Address for correspondence:

Dr. Mihai Mărginean

Department of Anaesthesia

Military Hospital

22 Traian Moşoiu St

400132 Cluj-Napoca, Romania

E-mail: marginean.m@gmail.com

presenting with this condition and the cardiovascular disturbances associated, a thorough examination and evaluation of the cardiovascular function is in order [2].

Recent studies have failed to demonstrate the role of minimally invasive monitoring in the management of septic shock patients for assessing the cardiovascular status. The conclusion reached was that focus ultrasonography should be promptly used in each case while the invasive hemodynamic monitoring is indicated in selected cases [3].

Multiple minimally invasive tools for assessing the hemodynamic profile have been developed in recent years, with most of them using either the pulse contour or the transpulmonary thermodilution techniques. The former can be easily and rapidly used, but with less
\end{abstract}


accuracy, while the latter gives more accurate results but in turn requires higher expertise and more time to set up and interpret, as demonstrated by numerous studies carried out in patients with different hemodynamic profiles $[4,5]$. Validation studies specifically designed for septic patients are still scarce, and we need to carefully acknowledge and critically appraise their benefits and limitations [6]. Furthermore, several devices and specific software using these techniques have been developed by different companies, each of them with its advantages and disadvantages.

The aim of this study was to assess which of these techniques is more appropriate to be used in septic shock, in patients on high doses of vasoactive therapy: the pulse contour Vigileo ${ }^{\mathrm{TM}}$, third generation software, or the transpulmonary thermodilution principle on the newly developed EV1000 ${ }^{\mathrm{TM}}$ clinical platform by Edwards Lifesciences.

\section{Methods}

Our study was designed to fully comply with the Declaration of Helsinki regarding ethical principles for medical research involving human subjects and was approved by the Ethics Committee of the University of Medicine and Pharmacy in Cluj-Napoca. Individual informed consents were obtained from the closest relative of the patients prior to commencing the data acquisition, as patients were unable to give consent.

For this study we used data recorded by the Vigileo $^{\mathrm{TM}}$ (FloTrac sensor, Edwards Lifesciences $\odot$, software version 3.06) and EV1000 ${ }^{\mathrm{TM}}$ (VolumeView sensor, Edwards Lifesciences $\mathbb{C}$, software version 1.5) monitoring platforms. Both monitoring devices were connected to the same patient as follows: Vigileo ${ }^{\mathrm{TM}} /$ FloTrac collected data from a cannula inserted in the radial artery and the EV1000 $\mathrm{TM} /$ VolumeView from a femoral artery. After performing an echography of the arteries (both radial and femoral) we decided which ones to be cannulated, according to the anatomy, blood flow and pathological findings, always aiming to cannulate an artery with a normal flow. Every patient had the Vigileo ${ }^{\mathrm{TM}}$ monitor set up first and the EV1000 device was added when the patient's clinical status would require it (no response to fluid loading and the need to increase the vasoactive therapy in order to maintain a MAP $>65 \mathrm{mmHg}$ ). No patient was subjected to unnecessary invasive maneuvers with the purpose of collecting data. After both monitoring devices had been in place, we started collecting data at the same time from the two of them. We obtained information from four critically ill patients with different ICU admission criteria (medical and surgical) that developed septic shock [7], and were receiving, at the time of data acquisition, vasoactive therapy in high doses (more than $0.6 \mathrm{mcg} / \mathrm{kg} / \mathrm{min}$ of norepinephrine), which was initiated after adequate fluid loading. The adequacy of fluid loading was determined by assessing the variation of the stroke volume on Vigileo ${ }^{\mathrm{TM}}$ monitor, while performing the passive leg rising (PLR); if there was less than $15 \%$ increase in SV while performing the PLR maneuver, we considered the patients a nonresponder to fluids.

Also, they were intubated and mechanically ventilated using tidal volumes of 4-6 ml $/ \mathrm{kg}$, in a pressure controlled ventilation mode (Bi-level Positive Airway Pressure, PEEP of $5 \mathrm{~cm} \mathrm{H}_{2} \mathrm{O}$, Inspiratory Pressure between 22-30 $\mathrm{cm} \mathrm{H}_{2} \mathrm{O}$ ). Analgesia and sedation were provided with a continuous infusion of fentanyl and propofol with a sedation score of 6 on the Ramsay sedation scale and no ventilator dyssynchrony.

When both monitoring devices were in place, time was synchronized between them, and data collecting started. Vigileo ${ }^{\mathrm{TM}}$ monitor does not need calibration, and the EV1000 ${ }^{\mathrm{TM}}$ platform was calibrated every 8 hours as well as after any vasoactive infusion modification following manufacturer recommendations. Data from two patients were recorded every 5 minutes for 12 hours (approx. 145 data entries) and for the other two, every 1 minute for 4 hours (approx. 240 data entries). The parameters included in the study were mean arterial pressure (MAP), stroke volume (SV) and cardiac output $(\mathrm{CO})$.

Statistical analysis was performed with IBM ${ }^{\circledR}$ SPSS ${ }^{\circledR}$ Statistics ver.22 (Armonk, NY, USA). The results were considered significant at the level alpha less than $0.05(\alpha=0.05)$.

Because neither of the two methods of assessing the hemodynamic status is a "gold standard" we cannot offer data about their accuracy. Due to the fact that a good correlation of data was obtained when the transpulmonary thermodilution technique was compared with the gold standard technique (the pulmonary artery catheter) in septic shock patients [8$10]$, and $\mathrm{EV} 1000^{\mathrm{TM}}$ device uses the aforementioned transpulmonary technique, we assumed this method to be the gold standard equivalent and considered any deviation of the Vigileo ${ }^{\mathrm{TM}}$ monitor away from the EV1000 ${ }^{\mathrm{TM}}$ values a deviation from the real value. We addressed the level of agreement between the two methods by visual examination of data patterns using graphs and studying the bias (the average deviation from the real value; the mean difference in values obtained with the two different methods), limits of agreement (lower limit, LLA; upper limit, ULA) and creating Bland-Altman plots $[11,12]$. In order to do that, two new variables (difference and mean) were computed for each parameter.

As a measure of systematic, proportional and random differences we computed a Passing-Bablock 
regression with the CUSUM test for linearity. These tests were performed using MedCalc for Windows, version 12.5 (MedCalc Software, Ostend, Belgium).

Because the Bland-Altman assumes a linear relation between errors and measurements, an assumption that is not defensible for measurements such as cardiac output (where, for example, a difference of 1 $\mathrm{L} / \mathrm{min}$ is more clinically relevant to low values as opposed to high CO values), we also computed the percentage error (proportion between the magnitude of measurement and the error in measurement which is calculated by dividing the confidence limit by the mean value of the measurements obtained with the established method - (ULA-LLA)/MeanEV1000x100). As recommended by Critchley and Critchley [13] an agreement between the two methods was evaluated by taking into account the possible errors of both methods. In clinical practice, an inaccuracy of up to $20 \%$ is regarded as acceptable. Comparing two methods each with an inaccuracy of $20 \%$ results in a maximum tolerable error of $28.28 \%$ (the combined inherent imprecision of both methods was calculated using this approach: (CV2 method $1+\mathrm{CV} 2$ method 2) 1/2; CV, coefficient of variation) which we approximated to $30 \%$.

It has been previously stated that inter-class correlation analysis (correlation coefficient, $r$; coefficient of determination, $r 2$ ) and comparing means ( $t$ test) offers insufficient and often inappropriate information regarding method agreement and such statistical methods were not employed [14].

\section{Results}

For a better description of the four patients included in this study we computed a table which includes demographic data, information regarding their pathology and basic physiological and ventilator parameters (Table 1).

For all the data sets the one sample $t$ test of the differences between the two methods against the null value was statistically significant meaning that the differences deviated from 0 (MAP, SV, CO p $<0.0001$ ). This is evident in the scatter plotting of the two sets of measurements against each other. An ideal agreement would position each dot on the $\mathrm{x}=\mathrm{y}$ axis (Fig. 1, 2 and 3).

We also computed graphs of SV, CO and MAP against time for both the methods used (Fig. 4). If we visually examine the graphs we can observe that for some of the time that data was collected, the trending between the two methods remained the same. There are periods of time in which the trending is lost, mostly related to medical interventions such as when norepi-
Table 1. Demographic data, pathological findings and basic physiological and ventilators parameters

\begin{tabular}{|ll}
\hline Demographic parameters & $\mathrm{N}(\%)$ \\
\hline Age & \\
$50-59$ & $2(50)$ \\
$60-69$ & $1(25)$ \\
$70-79$ & $0(0)$ \\
$80-89$ & $1(25)$ \\
\hline Gender & \\
\hline Male & $3(75)$ \\
\hline Female & $1(25)$ \\
\hline Pathophysiological findings & $\mathrm{N}(\%)$ \\
\hline Septic shock & $4(100)$ \\
\hline Surgical & $4(100)$ \\
\hline Oncological & $2(50)$ \\
\hline Non-oncological & $2(50)$ \\
\hline Without heart failure & $3(3)$ \\
\hline Heart failure NYHA I & $1(25)$ \\
\hline Arrhythmia & $0(0)$ \\
\hline ARDS & $1(25)$ \\
\hline Anemia & $4(100)$ \\
\hline Diabetes mellitus & $1(25)$ \\
\hline Acute renal failure & $3(75)$ \\
\hline Basic physiological parameters & Mean (SD) \\
\hline Heart rate & $85.96(36.77)$ \\
\hline Mean arterial pressure (MAP) & $73(12.76)$ \\
\hline Temperature ${ }^{\circ}$ C & $38.17(0.74)$ \\
\hline Sp $\mathrm{O}_{2}$ & $95.75(2.62)$ \\
\hline Basic ventilator parameters & \\
\hline Tidal volume ml/kgc & $4-6$ \\
\hline Pressure control ventilation N (\%) & $4(100)$ \\
\hline Positive End Expiratory Pressure mean (SD) & $6.25(1.82)$ \\
\hline Fraction of Inspired Oxygen mean (SD) & $0.45(0.14)$ \\
\hline & \\
\hline
\end{tabular}

nephrine or sedation infusions were changed. We mention that during the examined period we increased the infusion of norepinephrine in order to maintain a mean arterial pressure (MAP) over $65 \mathrm{mmHg}$, respectively $80 \mathrm{mmHg}$ in hypertensive patients. Some of the short periods of hypotension may be explained by the additional boluses of sedation which were administered before interventions such as tracheal suctioning to prevent complications.

Descriptive statistics and normality tests showed that the differences did not follow a normal distribution. This is also evident after inspecting the distribution graphs (Fig. 5, 6 and 7).

For this reason we computed Bland-Altman analysis as described in the Methods section for non-parametric data (Fig. 8, 9, 10 and Table 2).

The Passing-Bablock regression scatter diagram with the fitted regression line (and its 95\% confidence interval) as well as the identity $(\mathrm{x}=\mathrm{y})$ line are presented in Fig. 11, 12 and 13. 


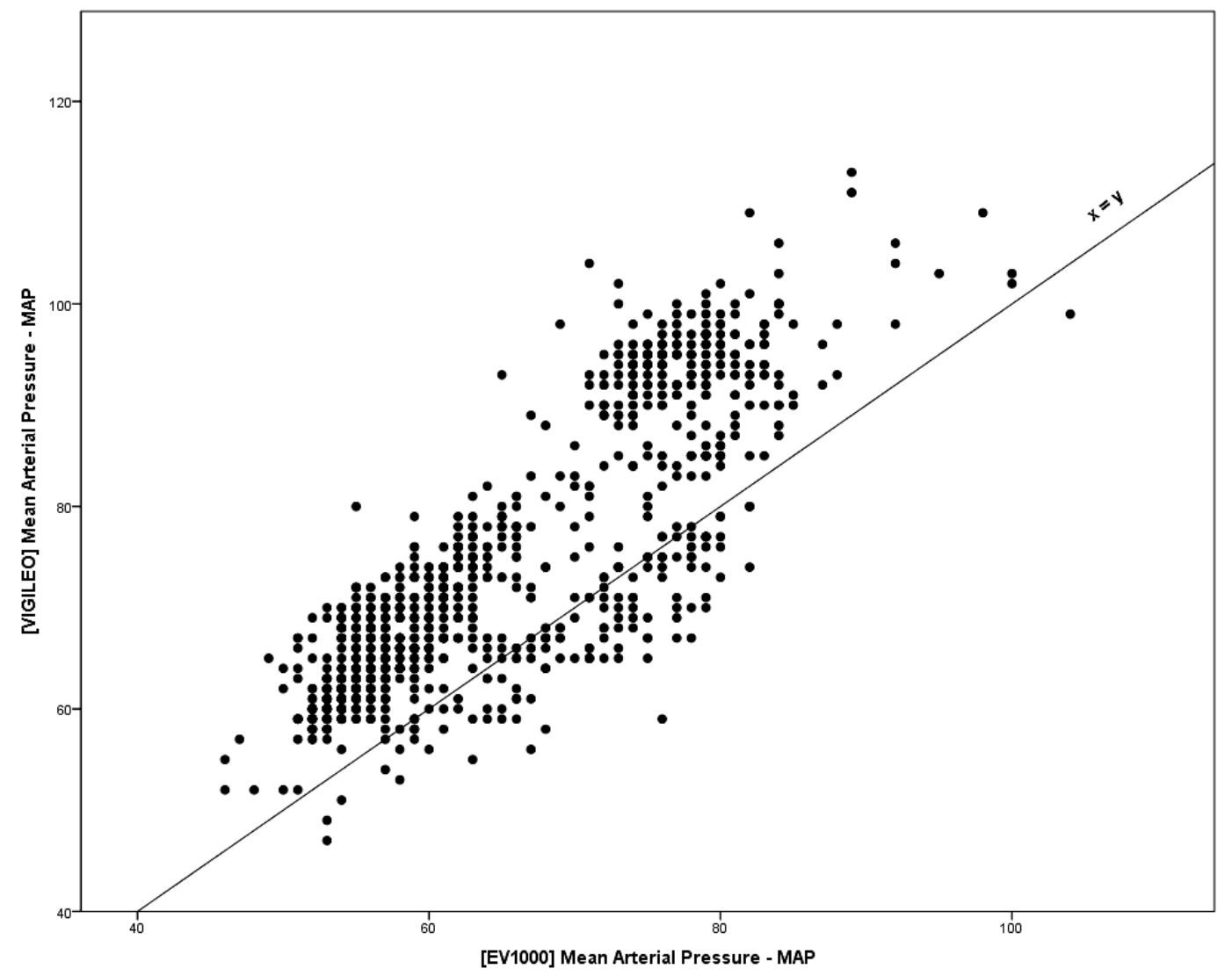

Fig. 1. Scatter plot of the Mean Arterial Pressure (MAP) values obtained with the two methods; the $x=y$ line is the line of equality

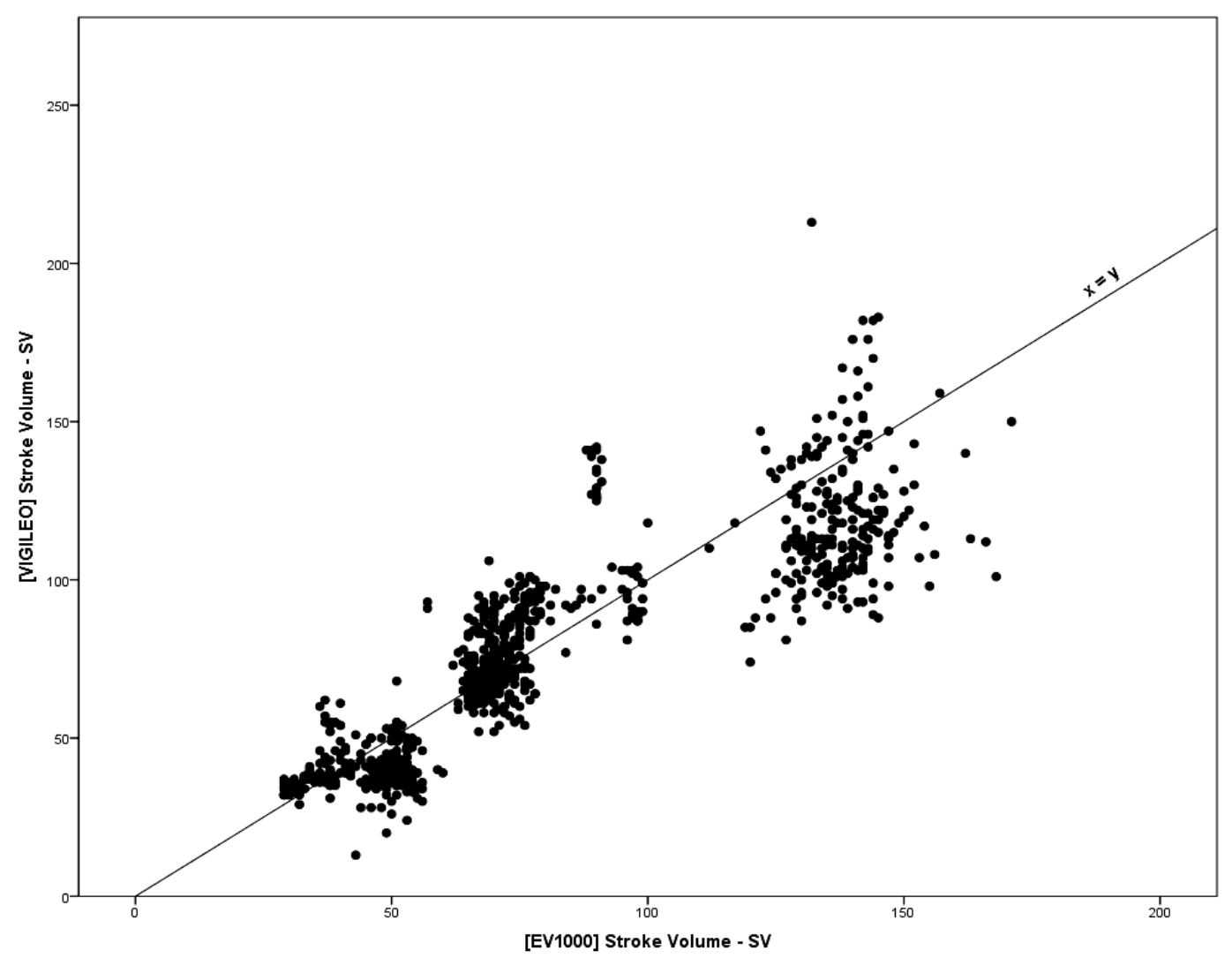

Fig. 2. Scatter plot of the Stroke Volume (SV) values obtained with the two methods; the $x=y$ line is the line of equality 


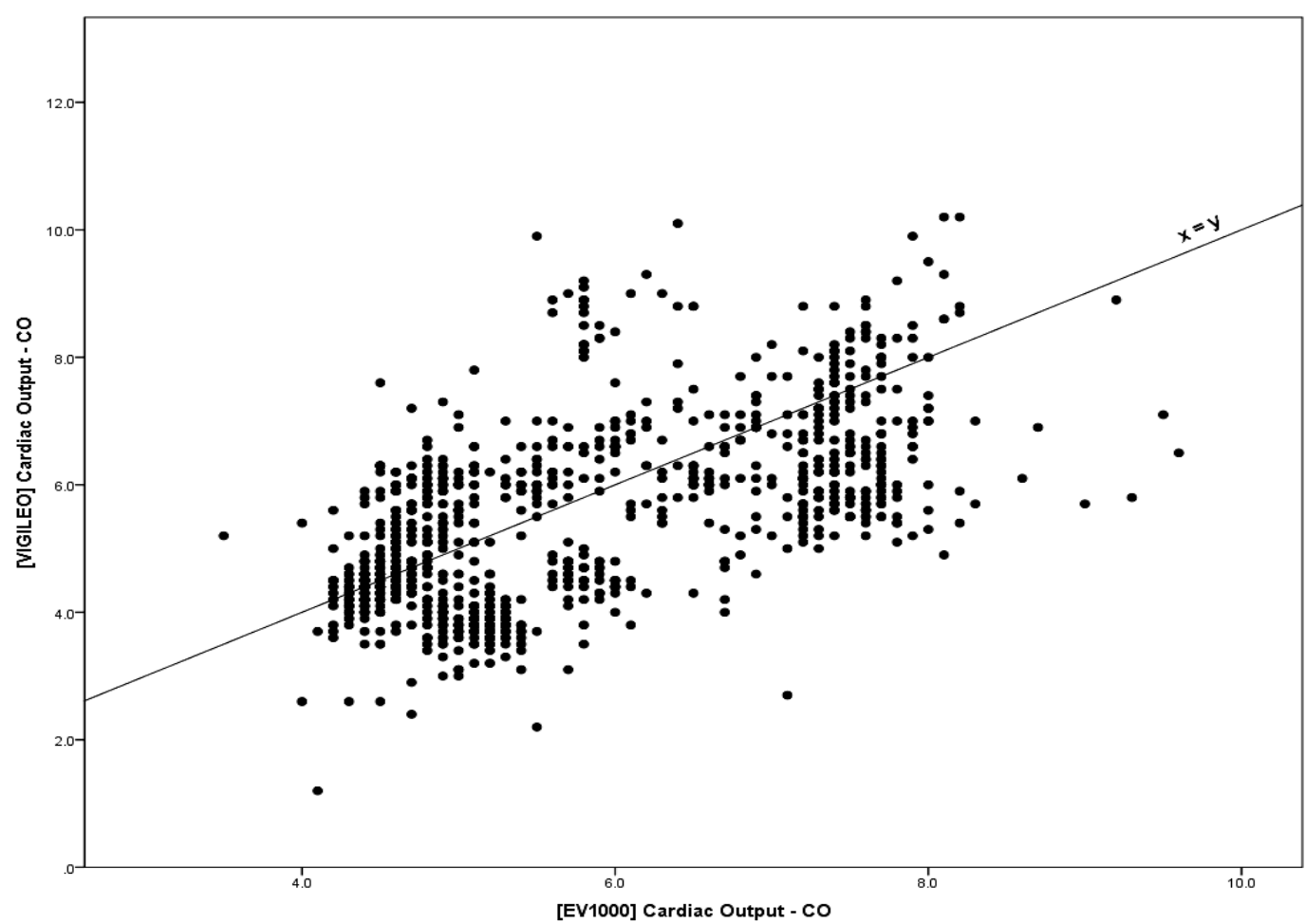

Fig. 3. Scatter plot of the Cardiac Output (CO) values obtained with the two methods; the $x=y$ line is the line of equality
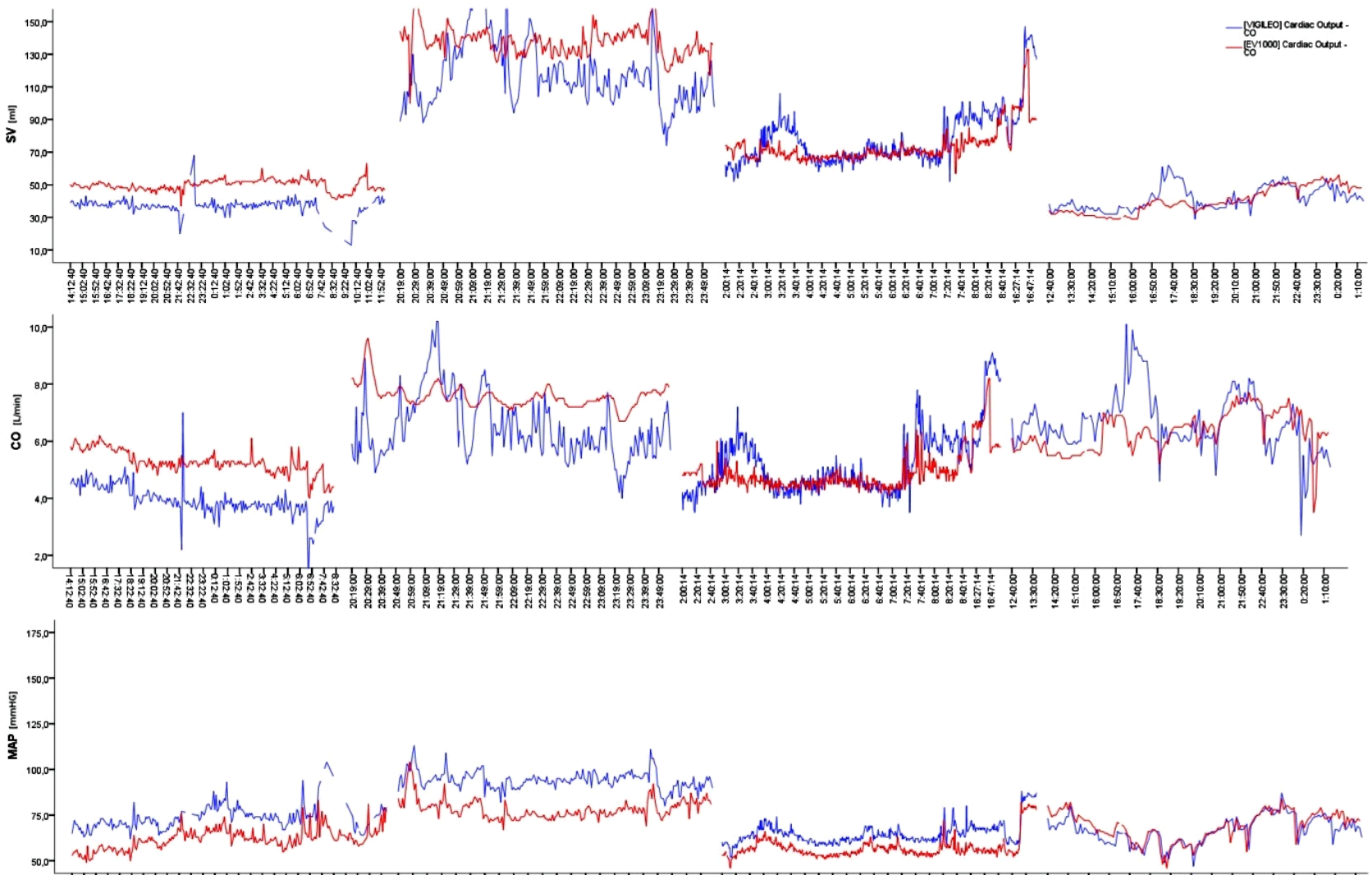

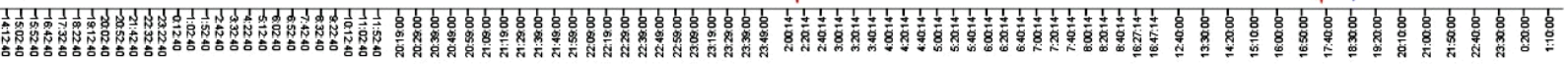
Time

Fig. 4. Graphical comparison of Stroke Volume (SV), Cardiac Output (CO) and Mean Arterial Pressure (MAP) values obtained with Vigileo and EV1000 methods 


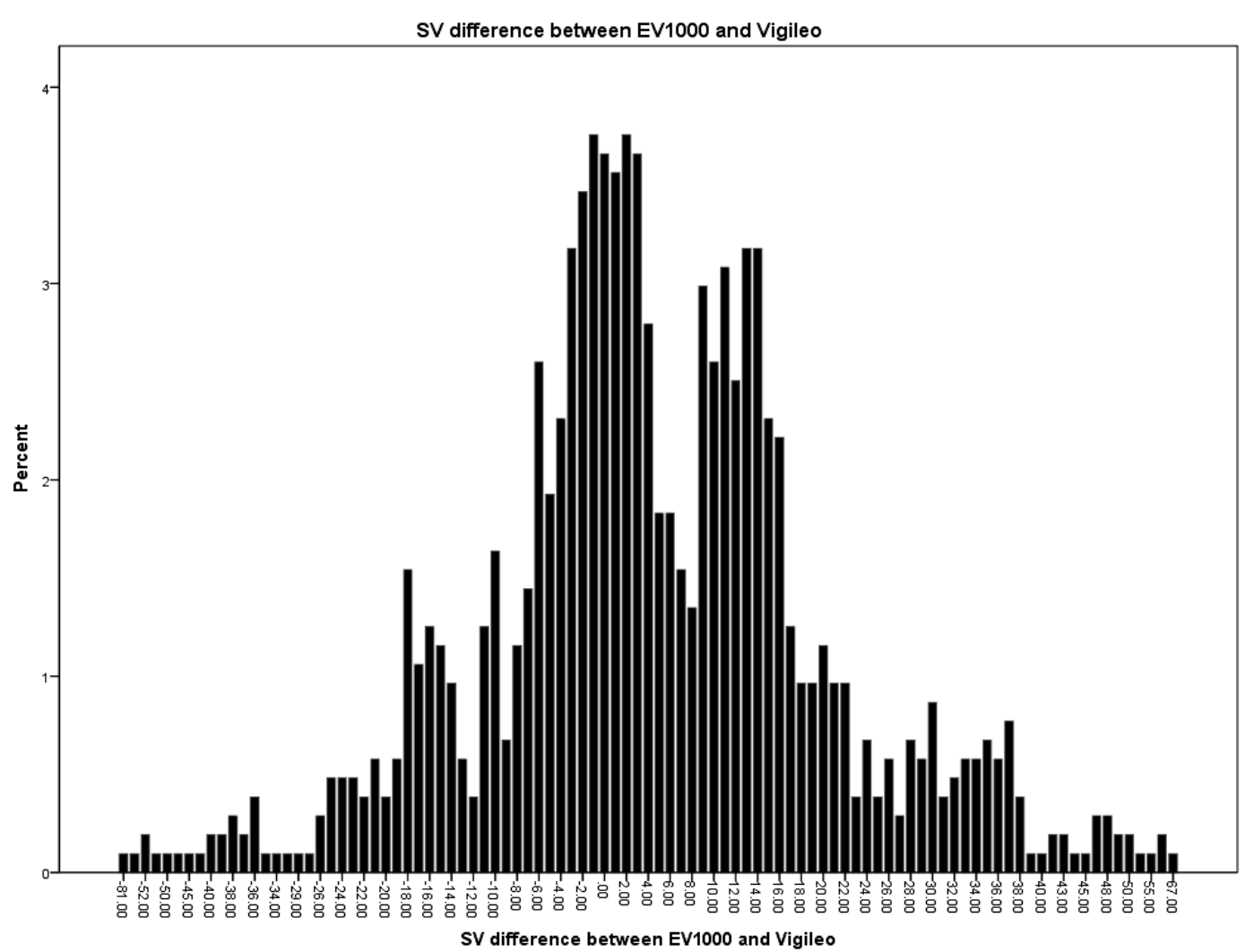

Fig. 5. The distribution of Stroke Volume (SV) differences between the Vigileo and EV1000 measurements

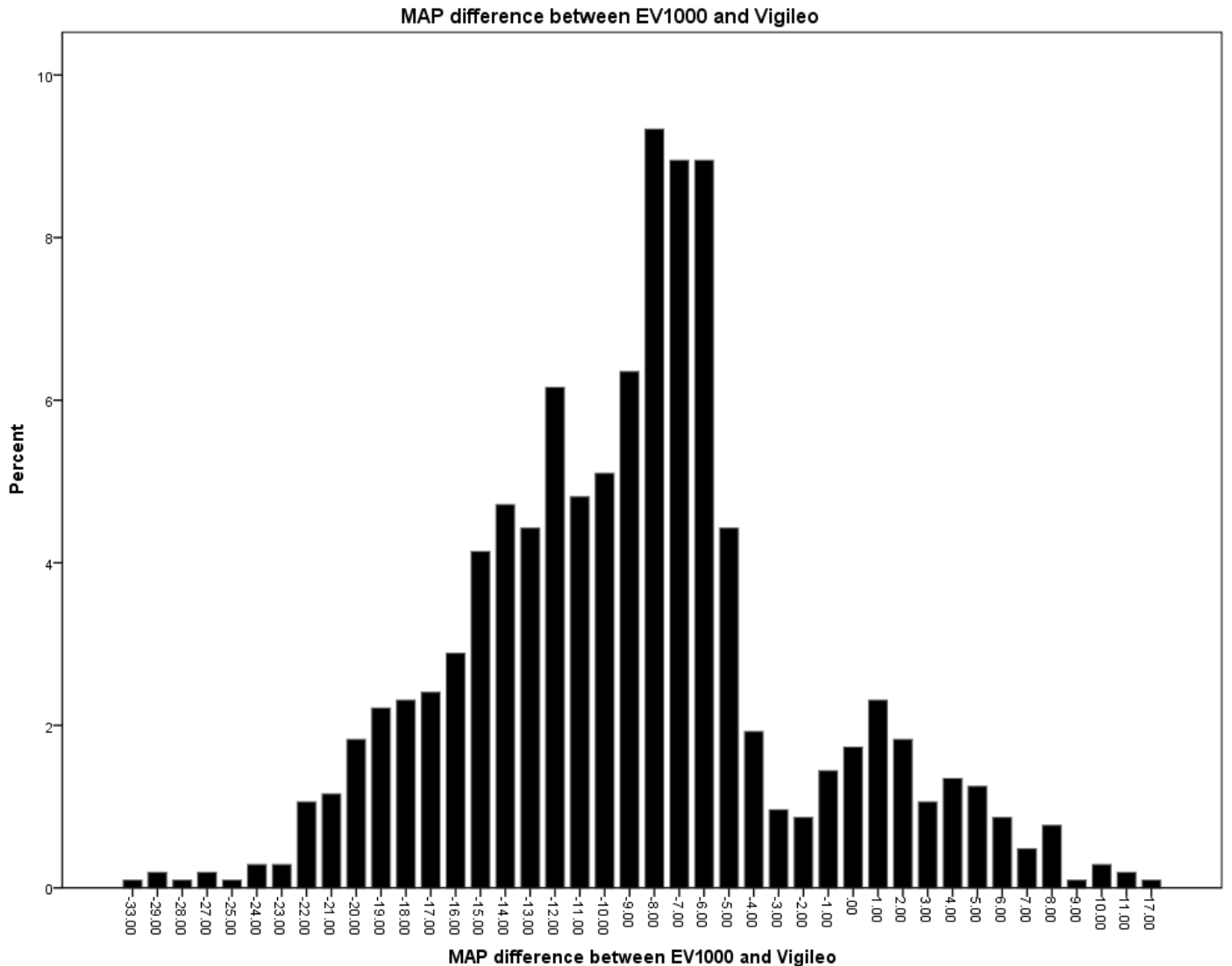

Fig. 6. The distribution of Mean Arterial Pressure (MAP) differences between the Vigileo and EV1000 measurements 


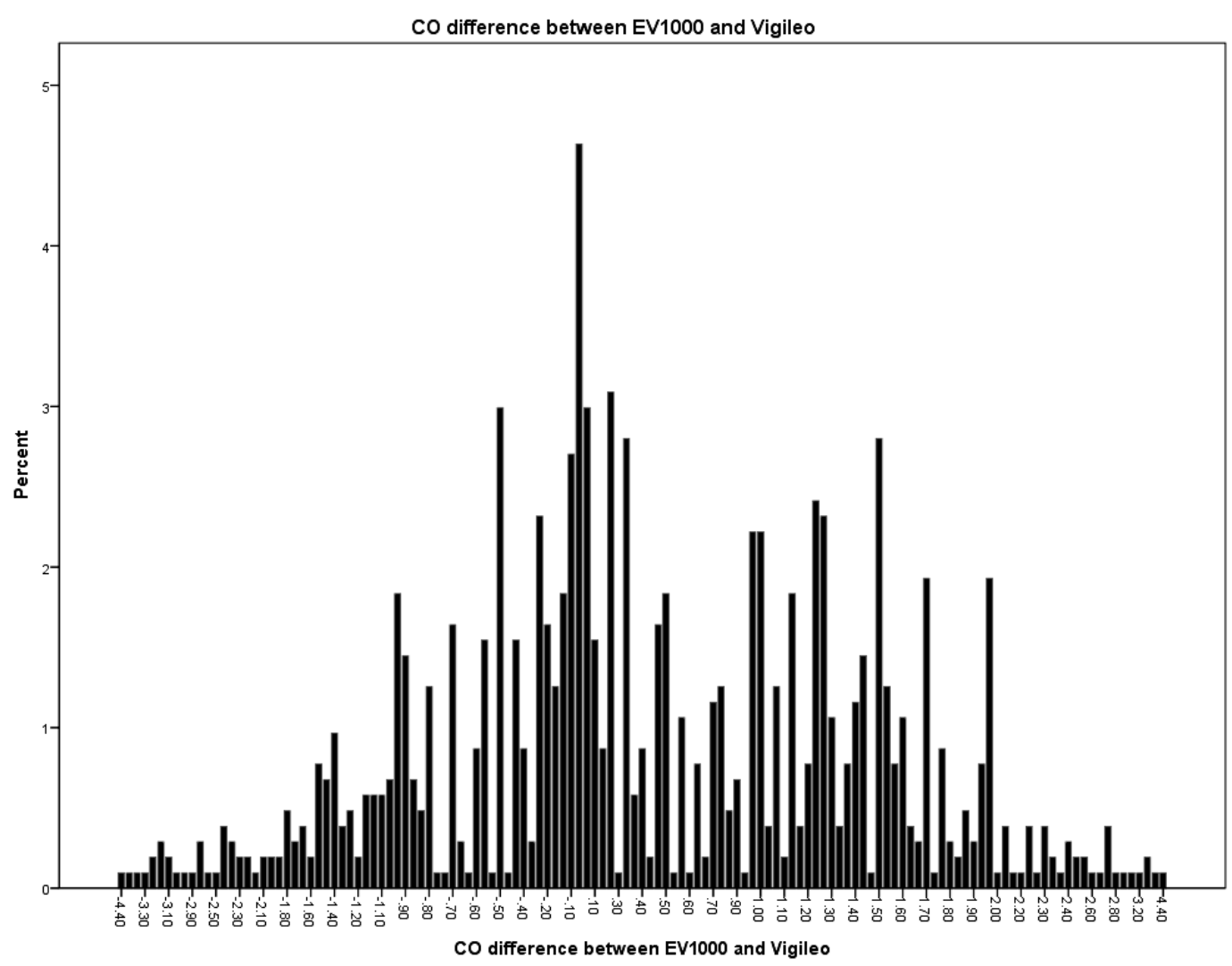

Fig. 7. The distribution of Cardiac Output (CO) differences between the Vigileo and EV1000 measurements

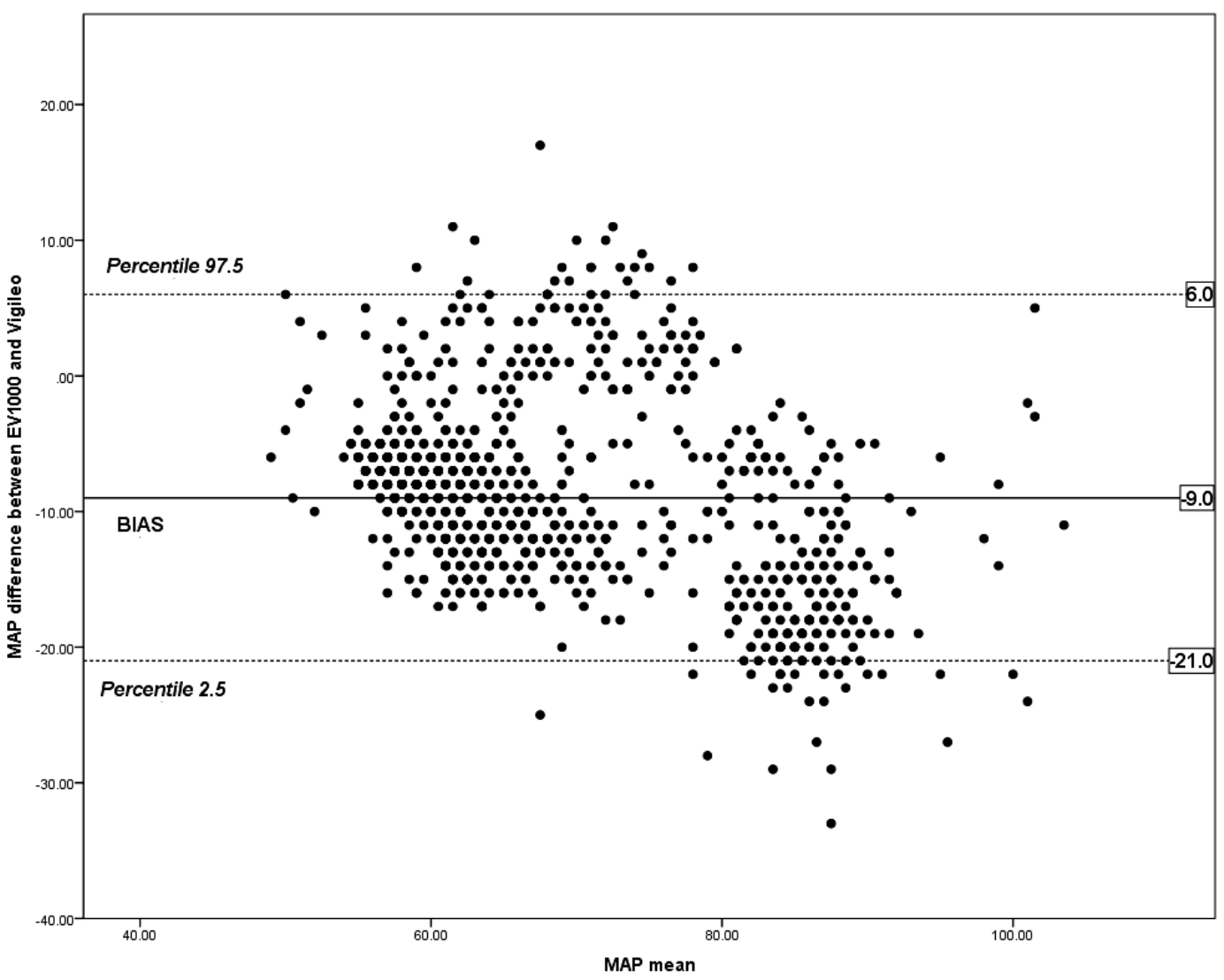

Fig. 8. Bland-Altman graph computed for Mean Arterial Pressure (MAP) 


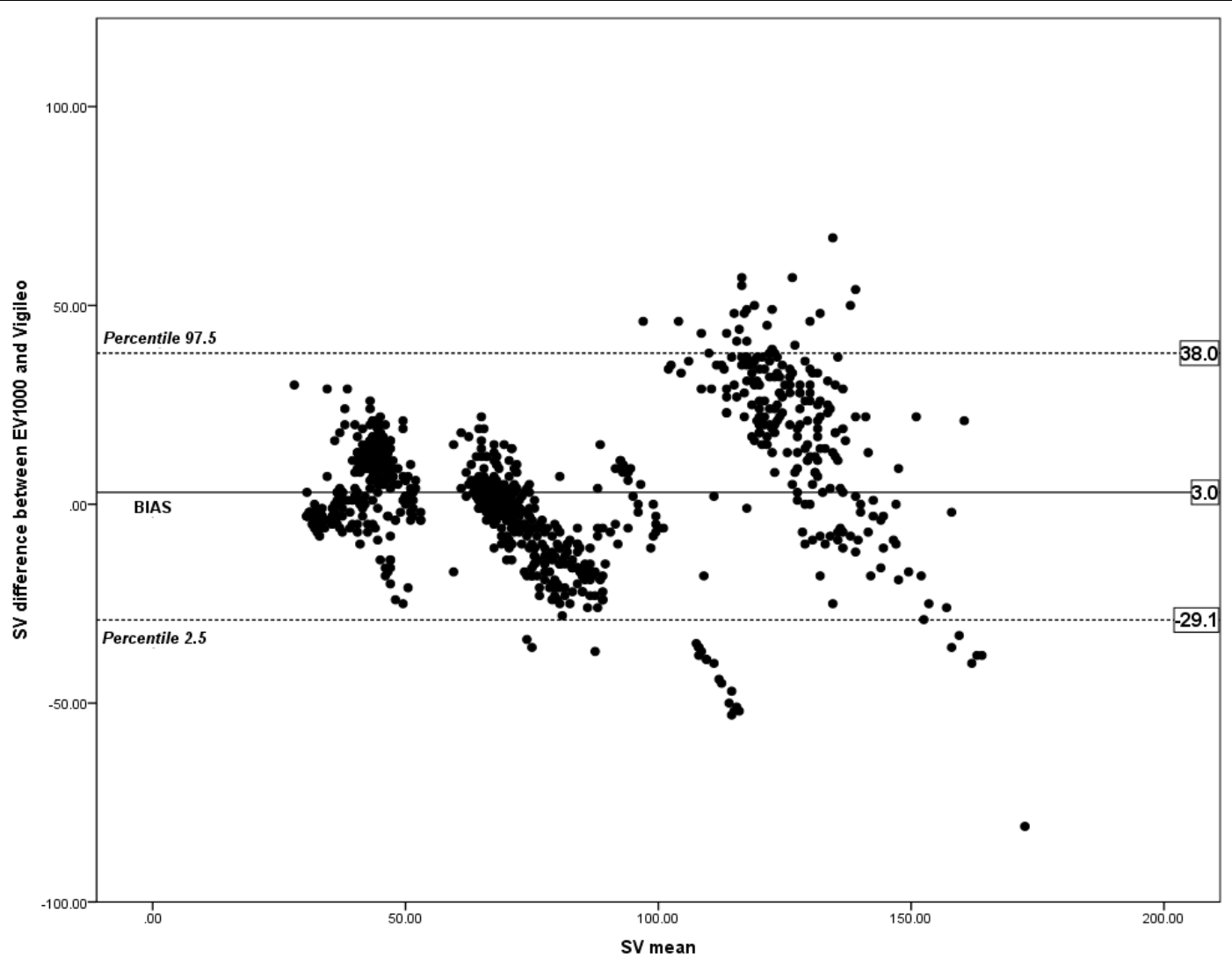

Fig. 9. Bland-Altman graph computed for Stroke Volume (SV)

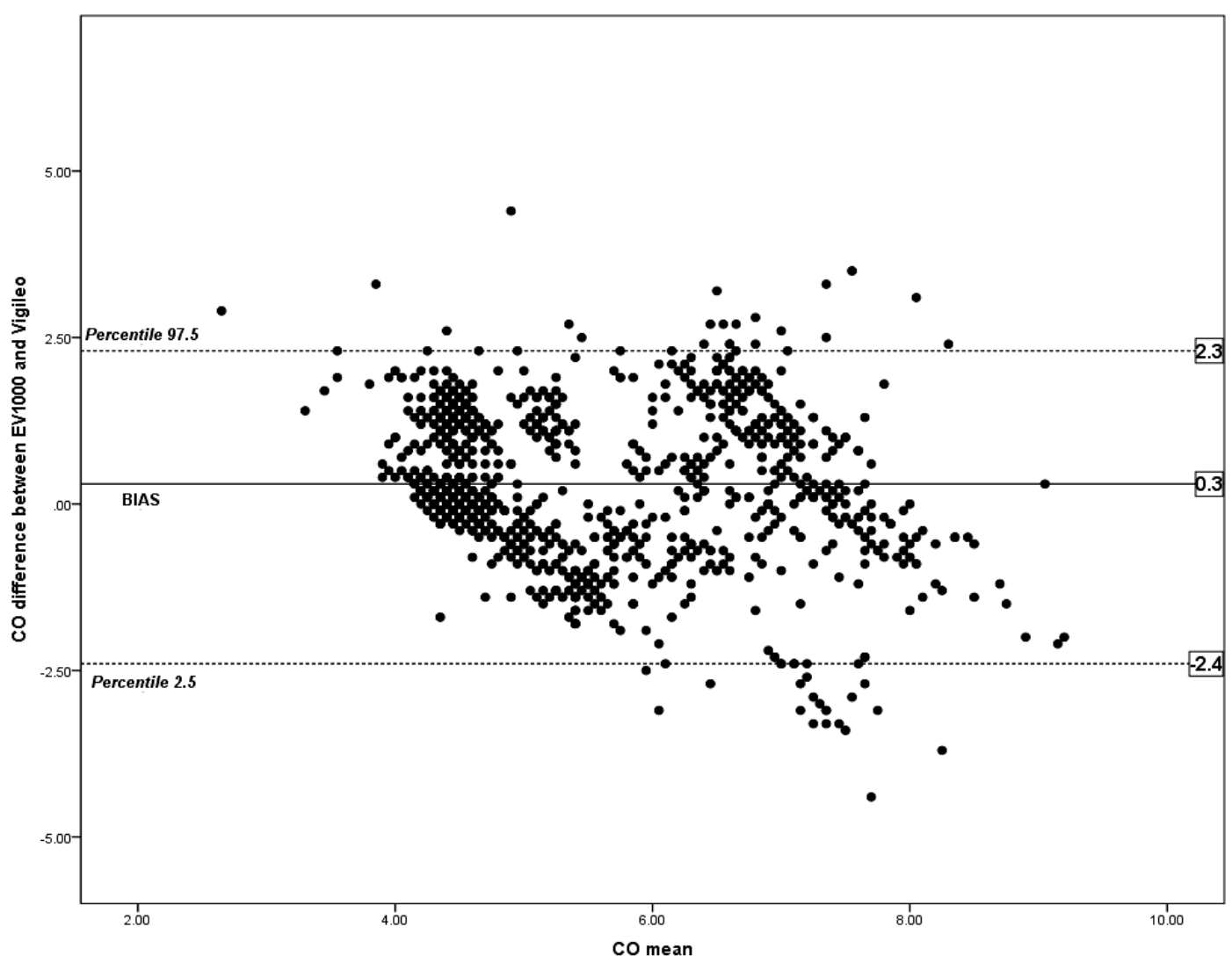

Fig. 10. Bland-Altman graph computed for Cardiac Output (CO) 


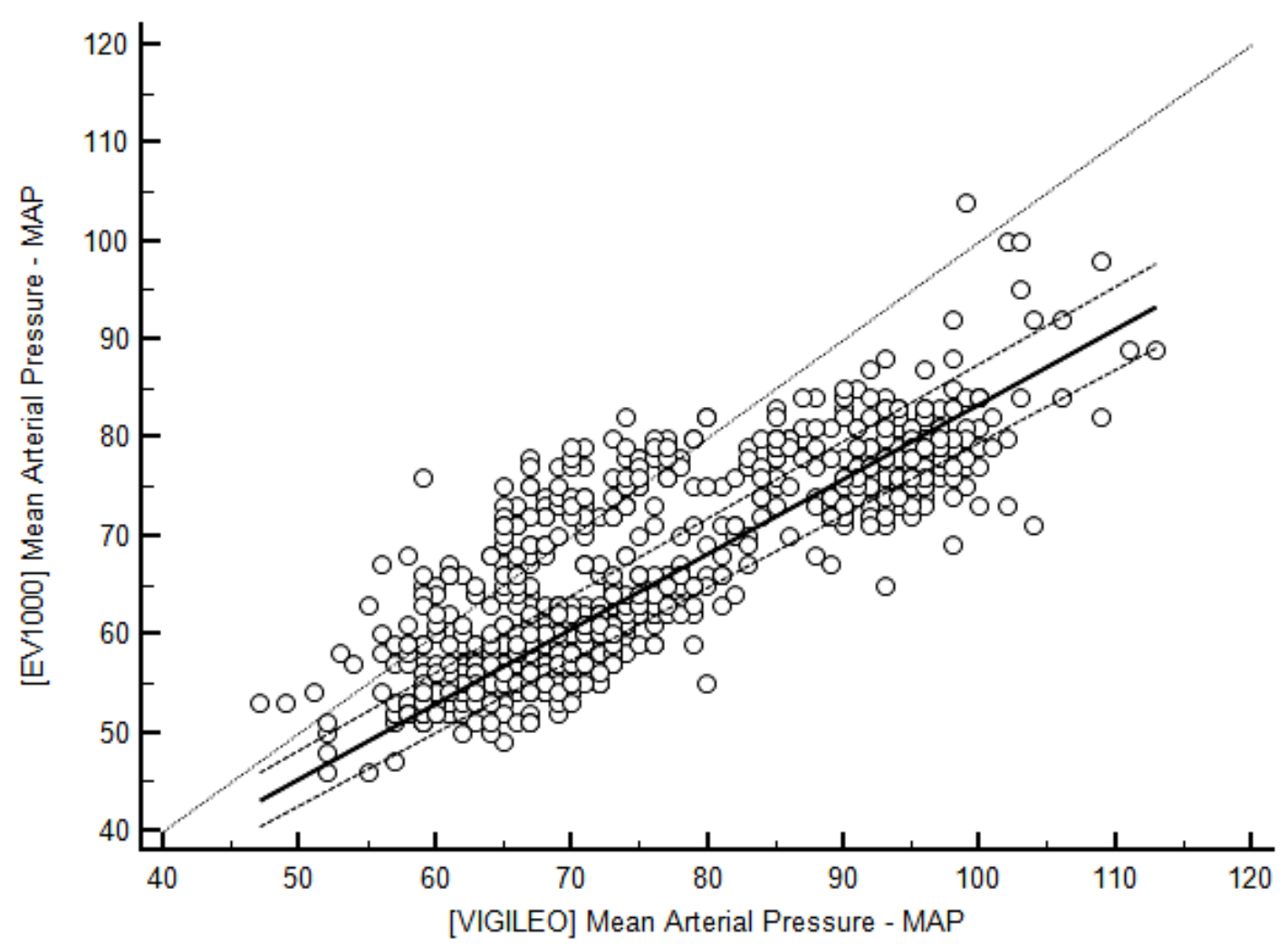

Fig. 11. Passing-Bablock regression analysis for Mean Arterial Pressure. Scatter diagram with the regression line (solid line), the confidence interval for the regression line (dashed lines) and identity line $(\mathrm{x}=\mathrm{y}$, dotted line)

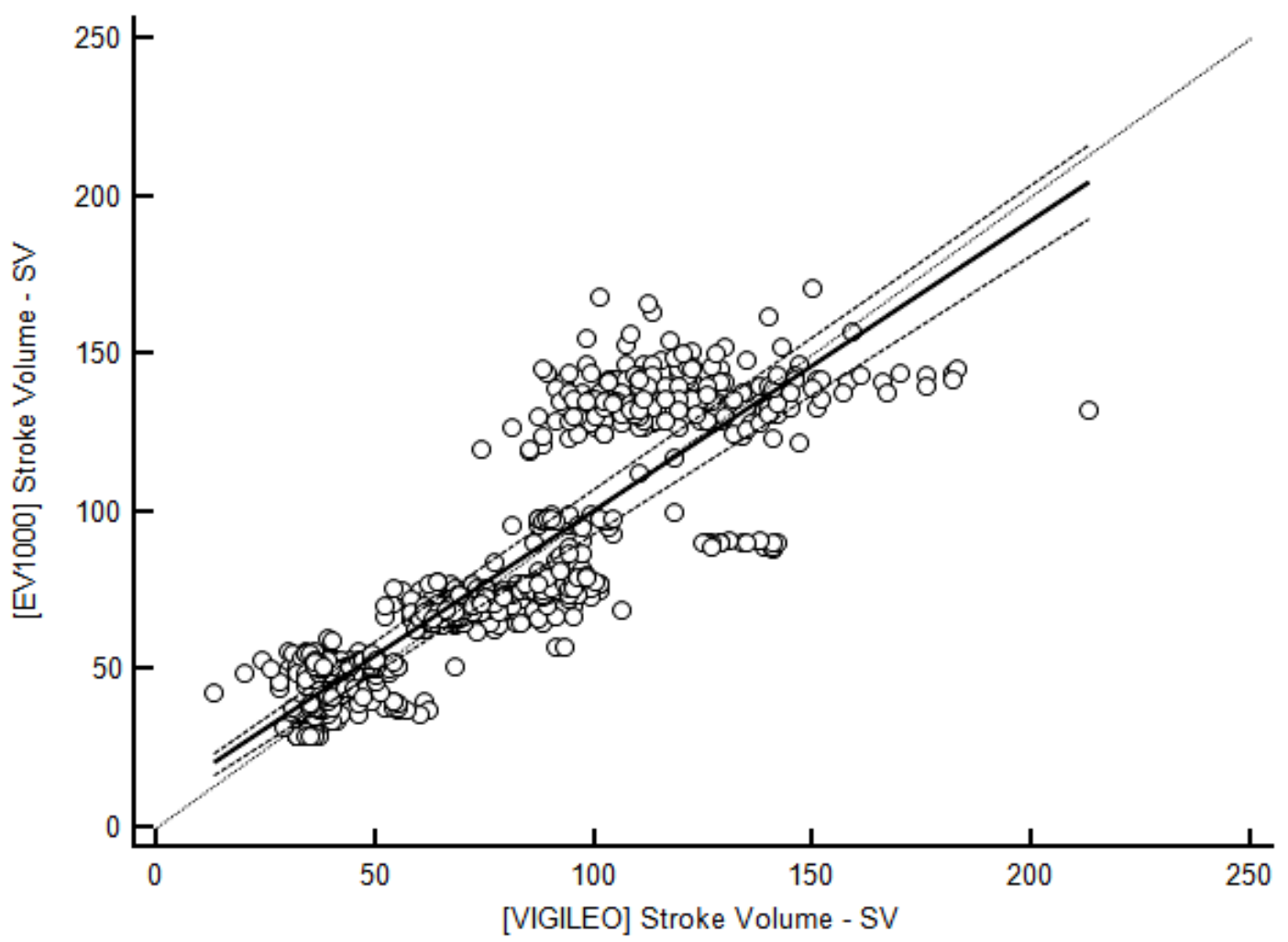

Fig. 12. Passing-Bablock regression analysis for Stroke Volume. Scatter diagram with the regression line (solid line), the confidence interval for the regression line (dashed lines) and identity line $(\mathrm{x}=\mathrm{y}$, dotted line) 


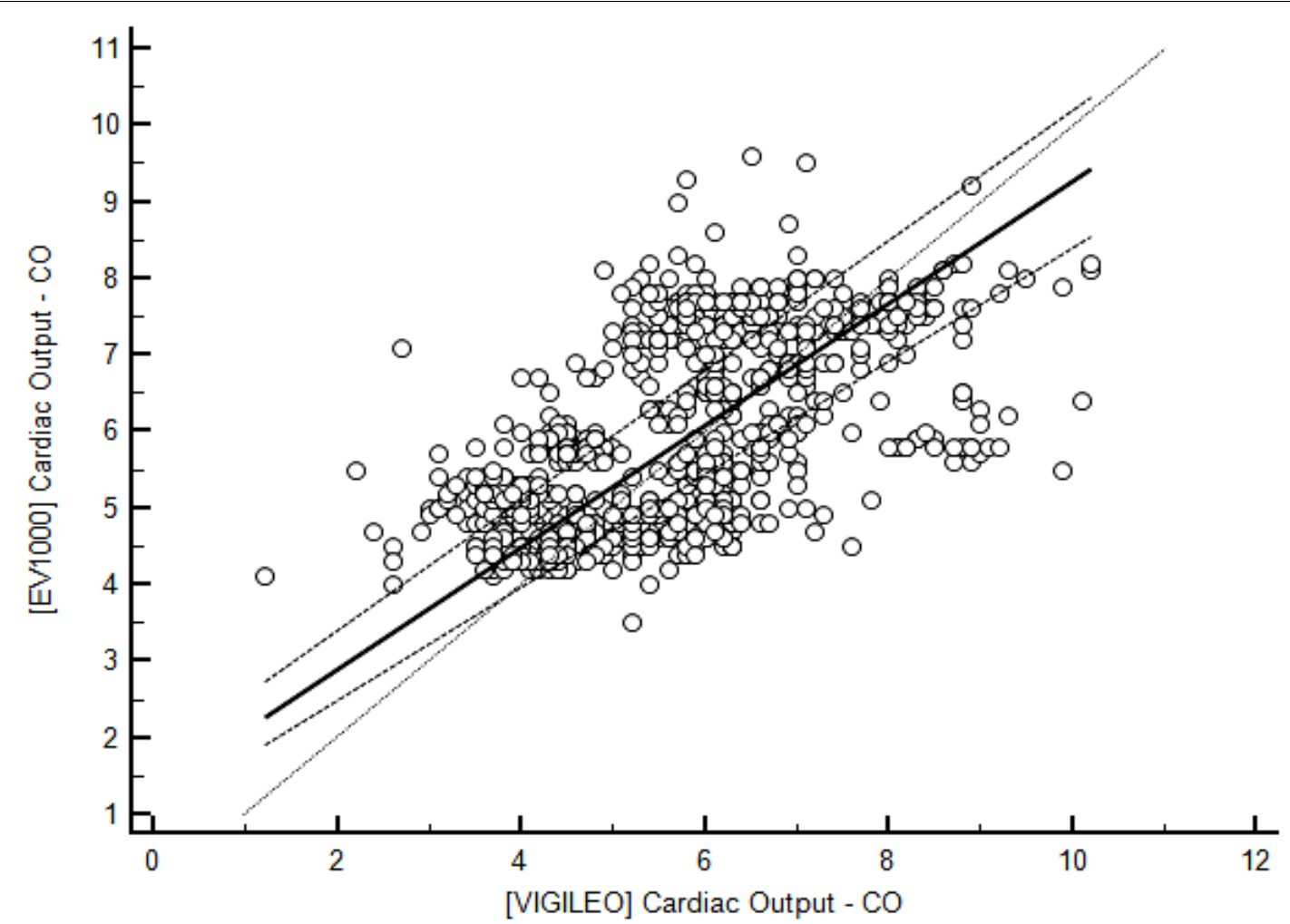

Fig. 13. Passing-Bablock regression analysis for Cardiac Output. Scatter diagram with the regression line (solid line), the confidence interval for the regression line (dashed lines) and identity line ( $\mathrm{x}=\mathrm{y}$, dotted line)

Table 2. Results from Bland-Altman analysis and Passing-Bablock regression

\begin{tabular}{|c|c|c|c|c|c|c|c|c|c|c|}
\hline \multirow{3}{*}{ Parameter } & \multicolumn{4}{|c|}{ Bland-Altman agreement } & \multicolumn{6}{|c|}{ Passing-Bablock regression } \\
\hline & \multirow{2}{*}{ bias } & \multirow{2}{*}{ LLA } & \multirow{2}{*}{ ULA } & \multirow{2}{*}{$\%$ error } & \multicolumn{2}{|c|}{ Systematic differences } & \multicolumn{2}{|c|}{ Proportional differences } & \multicolumn{2}{|c|}{ Random differences } \\
\hline & & & & & Intercept A & $95 \% \mathrm{CI}$ & Slope B & $95 \% \mathrm{CI}$ & RSD & $\pm 1.96 \mathrm{RSD}$ \\
\hline $\mathrm{MAP}[\mathrm{mmHg}]$ & -9 & -21 & 6 & 42.06 & 7.4 & $5.45-8.81 *$ & 0.76 & $0.74-0.78 * *$ & 5.62 & 11.01 \\
\hline $\mathrm{SV}[\mathrm{ml}]$ & 3 & -29 & 38 & 88.28 & 8.4 & $5.39-11.09 *$ & 0.92 & $0.87-0.96^{* *}$ & 16.4 & 32.14 \\
\hline $\mathrm{CO}[\mathrm{L} / \mathrm{min}]$ & 0.3 & -2.4 & 2.3 & 82.02 & 1.32 & $1.03-1.07^{*}$ & 0.79 & $0.73-0.85^{* *}$ & 1.03 & 2.01 \\
\hline
\end{tabular}

LLA = lower limit of agreement; ULA = upper limit of agreement; $\%$ error = percentage error; SV = stroke volume; CO = cardiac output; MAP $=$ mean arterial pressure; $\mathrm{CI}=$ confidence interval; $\mathrm{RSD}=$ residual standard deviation

* intercepts A CI does not contain the value 0 so we conclude that the methods might differ by at least a constant amount

** slopes B CI does not contain the value 1 so we conclude that there could be a proportional difference between the two methods

\section{Discussion}

All statistical tools that we employed were consistent in showing that the methods studied could not be used interchangeably. The Bland-Altman analysis revealed that the off-set between the measurements of SV could be as high as $67 \mathrm{ml}$ (ULA-LLA) which are clinically unacceptable. The percentage error for this amount of disagreement is also unacceptable if we use the Critchley criteria ( $\%$ error $<30 \%$ ) (Table 1$)$. Not surprisingly the $\mathrm{CO}$ measurements suffer from a similar level of disagreement. These findings were in accordance with most of the published literature regarding the minimal-invasive pulse-contour analysis devices such as the Vigileo/FloTrac monitor [15-17], but none of these studies compared devices produced by the same company, with similar software, in septic shock patients on high doses of norepinephrine.

In an article published in 2015, X. Monnet and J-L Teboul concluded that the uncalibrated devices become less reliable in the setting of rapid changes in vasomotor tone [18]. In 2016, in an article which was the result of an agreement between authors belonging to the Cardiovascular Dynamics Section of the European Society of Intensive Care Medicine, the conclusion was that uncalibrated systems become unreliable when major hemodynamic changes are occurring and these systems should be restricted to hemodynamically stable patients or when $\mathrm{CO}$ monitoring is required for short periods 
of time [19]. But there are many situations in which setting up a transpulmonary thermodilution system is either time consuming or contraindicated, and in such conditions using a pulse contour analysis cardiac output monitor can be beneficial, despite the errors that are statistically significant. Further research based on outcomes should be conducted to assess whether the clinical outcome is better when monitored with a less accurate cardiac output monitor, or without cardiac output motorization.

In a meta-analysis published in 2014 , it is stated that there is an improvement in the performance of the newer software of the uncalibrated FloTrac/ Vigileo $^{\mathrm{TM}}$, with a \% error at or below $30 \%$ in normoor hypodynamic conditions [20]. There is no data regarding the \% error of this new software in hyperdynamic states. In our study, we tested the agreement of the two methods in a subgroup of patients with septic shock on high doses of norepinephrine, and found no agreement.

One of the reasons for this disagreement between the $\mathrm{CO}$ and SV measured with the two methods might be due to the differences in MAP we found, in most cases, between the femoral and the radial sites, a difference which becomes greater with increasing doses of vasoactive drugs. The femoral-radial gradient was studied in an observational study of 24 critically ill patients and was found to be the highest in patients with septic shock and high infusion rates of vasoactive drugs [21].

The Passing-Bablock regression analysis tested positive for systematic differences. In theory, these differences could be corrected for and thus obtaining a better agreement. For our data set this could not be safely achieved because the linearity assumption was violated in all subsets, a possible reason for this being the small number of data points. Also, as could be observed by inspecting the Bland-Altman plots, the existing differences span on both sides of the bias line make thus correcting a systematic error very difficult. Furthermore, the proportional differences we have found could raise additional safety concerns. This means that the differences are not constant across the whole range of measurements but could be different at low levels as opposed to high levels making the interpretation of such measurements even more troublesome. We found that at low levels of CO and SV the differences were high, making the analysis of data at this critical level dangerous. These differences could be explained by the fact that low cardiac output states are associated with a higher systemic vascular resistances, and the $3^{\text {rd }}$ generation Flotrac/Vigileo was demonstrated to be less accurate in such states [4].

Even though we found statistical significant differences between the paired measurements obtained through the two techniques, we can say, by examining the trending graphs, that for some of the time that data was collected, trending between paired measurements was kept. More research is required in order to determine which conditions may disrupt the trending, and how much time is needed to reach the initial state after each intervention. Due to the fact that there were many interventions which were not recorded, we decided not to test the trending ability. We mention that during the examined period we increased the infusion of norepinephrine in order to maintain a mean arterial pressure (MAP) over $65 \mathrm{mmHg}$, respectively $80 \mathrm{mmHg}$ in hypertensive patients. Some of the short periods of hypotension may be explained by the additional boluses of sedation which were administered before interventions such as tracheal suctioning to prevent complications.

In spite of the large number of data sets, our study is limited by the small number of patients, making it difficult to extrapolate the results to the entire population. Keeping that in mind, our data shows a poor agreement between the two methods for hemodynamic assessment.

\section{Conclusion}

Even though some researchers have stated that the newer software version of the Vigileo/FloTrac is in better agreement with reference methods, our research which made use of the newest version to date (v3.06), found no agreement between data obtained with the two techniques in patients on high doses of norepinephrine. Further research is required in order to confirm these findings.

\section{Conflict of interest}

Nothing to declare

\section{References}

1. Gaieski DF, Edwards JM, Kallan MJ, Carr BG. Benchmarking the incidence and mortality of severe sepsis in the United States. Crit Care Med 2013; 41: 1167-1174. doi: 10.1097/CCM. 0b013e31827c09f8

2. Vincent JL. Safety considerations of septic shock treatment. Expert Opin Drug Saf 2016; 15: 215-221. doi: 10.1517/ 14740338.2016.1128411

3. Seymour CW, Rosengart MR. Septic Shock: Advances in diagnosis and treatment. JAMA 2015; 314: 708-717. doi: 10.1001/ jama.2015.7885

4. Monnet X, Anguel N, Jozwiak M, Richard C, Teboul JL. Thirdgeneration FloTrac/Vigileo does not reliably track changes in cardiac output induced by norepinephrine in critically ill patients. Br J Anaesth 2012; 108: 615-622. doi: 10.1093/bja/aer491

5. Hadian M, Kim HK, Severyn DA, Pinsky MR. Cross-comparison of cardiac output trending accuracy of LiDCO, PiCCO, FloTrac 
and pulmonary artery catheters. Crit Care 2010; 14: R212. doi: $10.1186 / \mathrm{cc} 9335$

6. Cecconi M, Arulkumaran N, Kilic J, Ebm C, Rhodes A. Update on hemodynamic monitoring and management in septic patients. Minerva Anestesiol 2014; 80: 701-711

7. Dellinger RP, Levy MM, Rhodes A, Annane D, Gerlach H, Opal SM, et al. Surviving sepsis campaign: international guidelines for management of severe sepsis and septic shock: 2012. Crit Care Med 2013; 41: 580-637. doi: 10.1097/CCM.0b013e $31827 \mathrm{e} 83$ af

8. Monnet X, Anguel N, Naudin B, Jabot J, Richard C, Teboul JL. Arterial pressure-based cardiac output in septic patients: different accuracy of pulse contour and uncalibrated pressure waveform devices. Crit Care 2010; 14: R109. doi: 10.1186/cc9058

9. Bendjelid K, Marx G, Kiefer N, Simon TP, Geisen M, Hoeft A, et al. Performance of a new pulse contour method for continuous cardiac output monitoring: validation in critically ill patients. Br J Anaesth 2013; 111: 573-579. doi: 10.1093/bja/aet116

10. Palmers PJ, Vidts W, Ameloot K, Cordemans C, Van Regenmortel $\mathrm{N}$, De Laet I, et al. Assessment of three minimally invasive continuous cardiac output measurement methods in critically ill patients and a review of the literature. Anaesthesiol Intensive Ther 2012; 44: 188-199

11. Bland JM, Altman DG. Statistical methods for assessing agreement between two methods of clinical measurement. Lancet 1986; 327: 307-310. DOI: http://dx.doi.org/10.1016/S01406736(86)90837-8

12. Hanneman SK. Design, analysis, and interpretation of methodcompariosn studies. AACN Adv Crit Care 2008; 19: 223-234. doi: 10.1097/01.AACN.0000318125.41512.a3

13. Critchley LA, Critchley JA. A meta-analysis of studies using bias and precision statistics to compare cardiac output measurement techniques. J Clin Monit Comput 1999; 15: 85-91. doi: 10.1023/ A: 1009982611386

14. Zaki R, Bulgiba A, Ismail R, Ismail NA. Statistical methods used to test for agreement of medical instruments measuring continuous variables in method comparison studies: a systematic review. PLoS One 2012; 7: e37908. doi: 10.1371/journal.pone. 0037908

15. Maxeiner H, Wenzel S, Weigand MA, Müller M. Cardiac output measured by the FloTrac/Vigileo system: Does the "plug and play" principle work? Appl Cardiopulm Pathophysiol 2010; 14: $131-138$

16. Biais M, Nouette-Gaulain K, Cottenceau V, Vallet A, Cochard JF, Revel $\mathrm{P}$, et al. Cardiac output measurement in patients undergoing liver transplantation: pulmonary artery catheter versus uncalibrated arterial pressure waveform analysis. Anesth Analg 2008; 106: 1480-1486. doi: 10.1213/ane.0b013e318168b309

17. Argueta E, Berdine G, Pena C, Nugent KM. FloTrac $₫$ monitoring system: what are its uses in critically ill medical patients? Am J Med Sci 2015; 349: 352-356. doi: 10.1097/MAJ. 0000000000000393

18. Monnet X, Teboul JL. Minimally invasive monitoring. Crit Care Clin 2015; 31: 25-42. doi: 10.1016/j.ccc.2014.08.002

19. Teboul JL, Saugel B, Cecconi M, De Backer D, Hofer CK, Monnet $\mathrm{X}$, et al. Less invasive hemodynamic monitoring in critically ill patients. Intensive Care Med 2016; 42: 1350-1359. doi: 10.1007/s00134-016-4375-7

20. Slagt C, Malagon I, Groeneveld ABJ. Systematic review of uncalibrated arterial pressure waveform analysis to determine cardiac output and stroke volume variation. Br J Anaesth 2014; 112: 626-637. doi: 10.1093/bja/aet429

21. Galluccio ST, Chapman MJ, Finnis ME. Femoral-radial arterial pressure gradients in critically ill patients. Crit Care Resusc 2009; 11: 34-38 